\title{
Agent Based Modeling of "Crowdinforming" as a Means of Load Balancing at Emergency Departments
}

\author{
Ryan Neighbour ${ }^{1}$, Luis Oppenheimer ${ }^{1,2}$, Shamir N. Mukhi ${ }^{1,3}$, \\ Marcia R. Friesen $^{1}$, Robert D. McLeod ${ }^{1}$ \\ ${ }^{1}$ University of Manitoba \\ ${ }^{2}$ Winnipeg Regional Health Authority \\ ${ }^{3}$ Public Health Agency of Canada
}

\section{Abstract}

This work extends ongoing development of a framework for modeling the spread of contacttransmission infectious diseases. The framework is built upon Agent Based Modeling (ABM), with emphasis on urban scale modelling integrated with institutional models of hospital emergency departments. The method presented here includes ABM modeling an outbreak of influenza-like illness (ILI) with concomitant surges at hospital emergency departments, and illustrates the preliminary modeling of 'crowdinforming' as an intervention. 'Crowdinforming', a component of 'crowdsourcing', is characterized as the dissemination of collected and processed information back to the 'crowd' via public access. The objective of the simulation is to allow for effective policy evaluation to better inform the public of expected wait times as part of their decision making process in attending an emergency department or clinic. In effect, this is a means of providing additional decision support garnered from a simulation, prior to real world implementation. The conjecture is that more optimal service delivery can be achieved under balanced patient loads, compared to situations where some emergency departments are overextended while others are underutilized. Load balancing optimization is a common notion in many operations, and the simulation illustrates that 'crowdinforming' is a potential tool when used as a process control parameter to balance the load at emergency departments as well as serving as an effective means to direct patients during an ILI outbreak with temporary clinics deployed. The information provided in the 'crowdinforming' model is readily available in a local context, although it requires thoughtful consideration in its interpretation. The extension to a wider dissemination of information via a web service is readily achievable and presents no technical obstacles, although political obstacles may be present. The 'crowdinforming' simulation is not limited to arrivals of patients at emergency departments due to ILI; it applies equally to any scenarios where patients arrive in any arrival pattern that may cause disparity in the waiting times at multiple facilities.

Keywords_Contact Graphs, Agent Based Models, Infection Spread Models 


\section{Introduction}

The worldwide H1N1 influenza pandemic (pH1N1) in 2009 and 2010 has mobilized and renewed research attention to the many facets of infection control and impact, ranging from the epidemiology of the illness, development and deployment of vaccines and other pharmaceutical interventions, and public health and emergency management measures. Predicting how an infection may spread within a population and the consequent impact that it may have includes forecasting with intelligent models and real data, as well as back-casting based on available data as one validation process. The focus of this paper is to present a computer simulation framework (model) of an urban community to model the spread of $\mathrm{pH} 1 \mathrm{~N} 1$ in the community, in which the model carries the capacity to model the impact of various intervention strategies, e.g. temporary clinics, vaccination, chemoprophylaxis, hygienic and social distancing measures. The intervention of direct interest in the present study is to provide the public with additional information related to expected waiting times at regional hospital emergency departments (EDs), and its potential impact on patient loads and consequent service delivery. A true metric associated with estimating expected wait times at a particular hospital is a random variable of many factors and difficult to estimate even with extensive ED data. The work here demonstrates the role that patient redirection can play if this metric were available. In the interim, we have associated the number of patients with a 'busyness' metric that would be amenable for presentation to the community. As the social dynamics and agent behaviours coupled with real data (economic, cultural, and social) become better defined, the computer simulation naturally allows one to focus on the population subsets and apply the framework to other jurisdictions.

The work is located within the larger context of healthcare informatics. The role of informatics in healthcare re-engineering and optimization has become a new reality in efforts to improve service delivery and efficiencies in healthcare. Both well vetted engineering approaches as well as emerging methods are being applied to generate solutions for health policy and decision makers. New service delivery paradigms are developed and justified from a variety of domains, including statistics, operations research and lean manufacturing concepts from industrial engineering, and business models [1]-[5]. In healthcare, the challenge is exacerbated by the inherent unpredictability of social behaviour; this contributes to the computational irreducibility of the problems encountered within many healthcare environments. As a consequence, modeling and simulation are playing a larger role as a design aid or tool in support of decision making [6][9].

\section{Background}

The computer simulation framework is built, in part, on an Agent-Based Model/Modeling $(\mathrm{ABM})$ approach. A central premise of this work is that ABMs - combined with real data and as high a resolution (fidelity) as the computer system affords - will create a new paradigm for a better understanding of epidemiology within a social system dynamics, and thereby lead to more effective tools for policy makers guiding the future. ABM is a relatively new approach to disease modeling (10+ yrs), an area historically addressed by well-vetted mathematical modeling techniques $(70+\mathrm{yrs})$. However, the use of ABM for simulating infection spread within an urban area and built upon the incorporation of real data is only now emerging (2+yrs). ABM is based on simulating a collection of agents - i.e. the people in the model - in terms of their 
characteristics, behaviours, and interactions with other agents. Agents (people) are purposeful and autonomous entities able to assess their situations, make decisions and compete with one another. ABM's conceptual depth is derived from its ability to model emergent behaviour that may be counterintuitive, and to discern a complex behavioural whole that is greater than the sum of its parts. Counterintuitive or counter intent behaviour may arise from non-obvious non-linear feedback loops that may exacerbate system dynamics, such as financial incentives to remain working while infected, or non-obvious pressures within the healthcare system that strain the system to its breaking point. Complex behaviour that is greater than the sum of its parts may be associated with an agent's ability to learn or make heuristic-based adaptations to their behaviours. ABM provides a natural description of a system that can be calibrated and validated by representative expert agents (healthcare specialists), and is flexible enough to be tuned to high degrees of specificity (sensitivity) in agents' behaviours and interactions. ABMs are particularly well suited to system modeling in which agent behaviour is complex, non-linear, stochastic, and may exhibit memory or path-dependence [10][11][12]. Early application areas of ABM include logistics, economics, and transportation systems.

ABM also provides one of the most useful tools available in terms of knowledge transfer and requirements capture, independent of whatever other techniques may also be employed. The model construction forces ideas to be clarified; unclear and hidden assumptions are exposed and debated in a common and familiar lexicon, leading to the ABM having a direct correspondence to the problem as understood by the practitioner and the developer. The resulting model closely resembles the system description, which could come from business rules or some other description by stakeholders who need not be overly familiar with ABM itself. Many ABMs are developed to gain a better understanding of operations through the use of what-if scenarios, and in doing so will provide a decision support tool to public health decision-makers.

A more recent and considerable area of application for ABMs has been country scale (community-level) disease spread modeling in human populations [13][14]. The focus generally constitutes large scale community-level epidemics of respiratory infections, as this is an important public health and policy issue with far-ranging health and economic impacts. Our own work has included the development of one of the first urban-level epidemic proof-of-concept ABMs, based on a paradigm of a 'discrete space scheduled walker' (DSSW) [15]. The urban scale ABM is one of the most appropriate modeling levels in terms of incorporating high resolution data (individual based), as well as for simulating social dynamics reinforced by patterns of behaviour and readily available topographical data. The proof-of-concept is built on synthesized data and a very limited range of agent parameters. It can model a medium-sized North American City using approximately 650,000 discrete agents (people), each of whom are assigned a demographic profile and a weekly schedule on the topography of the City of Winnipeg, Canada. Running the simulation with these types of numbers however is computationally tasking for a reasonable desktop environment. The proof-of-concept was built upon a conceptual framework of statistical reasoning (law of large numbers, statistical mechanics) as well as a correct-by-construction bias, meaning that the system dynamics emerge directly out of the agents' characteristics and behaviours, rather than by the inclusion of artificial small-world networks. The proof-of-concept addresses where, who, when, and what elements. Where: underlying topographical (network / graph) data is extracted from map and search engine utilities such as Google Earth, in order to build a network of objects, denoted institutions. 
Institutions are existing geographic locations such as homes, businesses, schools, leisure sites, hospitals, airport, and transport vehicles. Who: Agents are people that make up a community, and between whom an infection would be spread. When: a central premise of the DSSW-ABM is that agents are primarily creatures of habit, operating on routine schedules with slight random perturbations [16]. What: The factor of interest is the spread of the $\mathrm{pH} 1 \mathrm{~N} 1$ virus or other viruses that cause respiratory infections among the population of agents, through agent-to-agent contact associated with routine daily activities. Once infected, the agents probabilistically choose to stay home for the duration of the infection or attend a hospital ED.

As an intervention, we have considered modeling the potential role of 'crowdinforming' in directing or governing an agent's behaviour. The notion of 'crowdinforming' is a natural extension of 'crowdsourcing' [16], whereby data collected by crowdsourcing is again fed back to the 'crowd' via public access. Low-tech examples of the role of 'crowdinforming' in modifying human behaviour are readily available, such as line-ups at vaccination clinics as vaccine shortages are announced in the mass media. While a fairly obvious intervention, 'crowdinforming' is a novel inclusion into the ABM and simulation.

\section{Methods}

The ABM engine is coded in $\mathrm{C}++$, an Object Oriented Language. The object oriented approach has natural extensions to the spatial modeling inherent in the spatial nature of the system under study. Developing an ABM within an object oriented framework from the ground up provides an additional degree of understanding the problem in contrast to using a more commercial platform. The simulated world is a two-dimensional $(\mathrm{X}, \mathrm{Y})$ discrete Cartesian world of extremely high resolution. Building upon the proof-of-concept, the ABM is general purpose and at present is a spatially directed ABM reflective of a specific topography - in this case, the City of Winnipeg, Canada. Agents can access a limited number of features of other agents and objects, which can be set by the programmer, depending on the model. They can also pass messages to other agents in order to achieve interaction. The ABM houses institutions such as hospitals, homes, malls, leisure facilities, schools, businesses, and transportation institutions such as cars and public transport. Graphical inputs serve as defining location derived from maps and community planning documents. The ABM framework is illustrated in Fig. 1. The publicly accessible information 'dashboard' is illustrated on the left-hand side, providing near real time information on waiting times at various EDs at hospitals within a region. Currently, this information is available in individual facilities' waiting rooms; yet, for the present simulation it is assumed to be web accessible to the general public via readily available web services. The web services can include traditional web 2.0 applications supporting dynamic updating of web pages based on new information from various facility waiting time estimates, as well as access via traditional wired services to those offered over $3 \mathrm{G}$ cellular networks or 'smart phones'. 


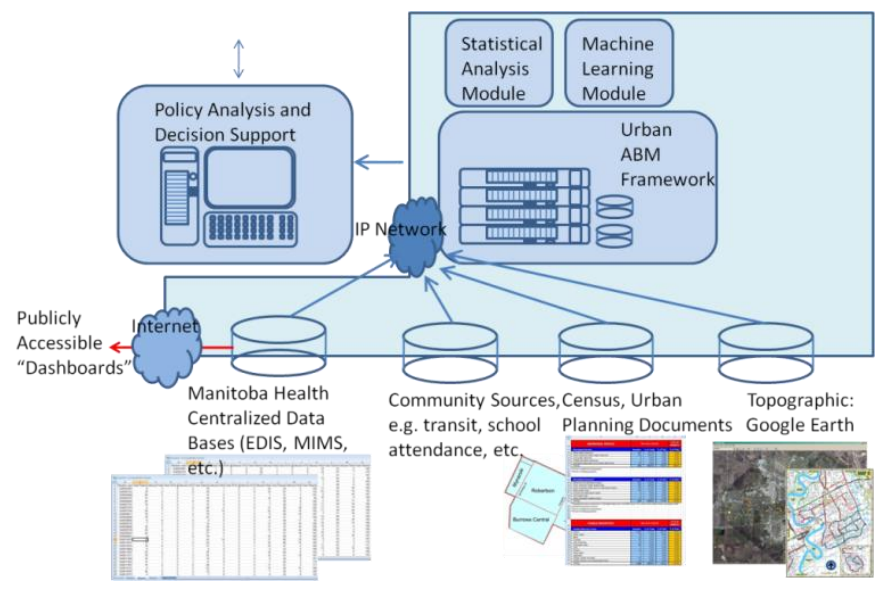

Fig. 1. The ABM framework encompassing various data sources.

\section{A. Modeling Surges at Hospitals}

This section outlines the urban based ABM under conditions associated with an ILI outbreak in Winnipeg, Canada. In the model, a parameterized distribution of households is associated with a colour as a graphical input. For this scenario, various colours represent a number of multiple person households uniformly distributed across Winnipeg, as shown in Fig. 2. At this scale, the colours tend to blend although roads, rivers, and other infrastructure and features are clearly evident.

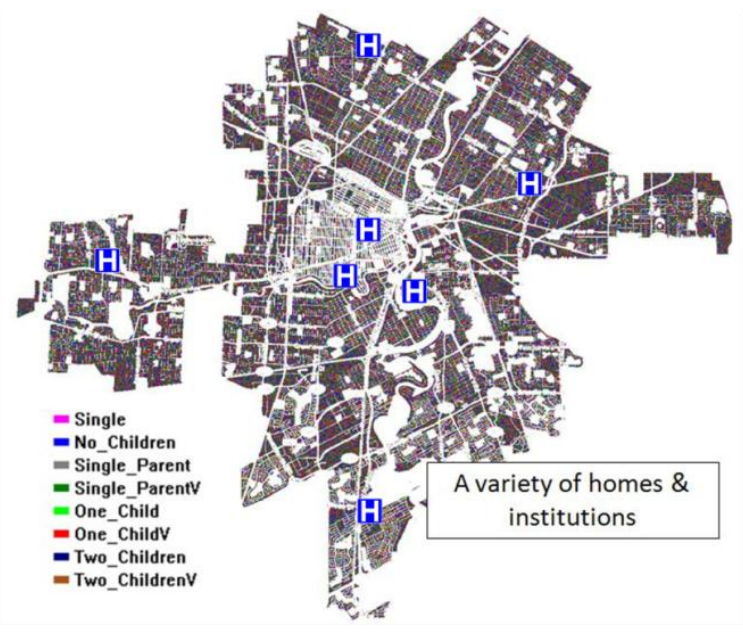

Fig. 2. A screenshot of the ABM input.

A number of institutions such as schools, large businesses, restaurants, leisure institutes as well as hospitals are modelled. The spread of infection is a stochastic process with the probability of infection being directly related to social contact. Social contact can take place at home, work, school, or on public transport, with institutions also having probabilities of contraction associated with them. A baseline simulation involves scheduled agents being modelled, and as they become ill, they probabilistically go to the closest ED. Once at the ED, another individual-based state or phase model is introduced as illustrated in Fig. 3. At the ED, the agent may be discharged, 
treated, or admitted. Once admitted, the patient may again undergo treatment and recover, or alternatively the agent may not recover. The current institutional ED model is quite simple but could be extended and refined with a more detailed model as required, an example of which has been developed by the authors [18][19].

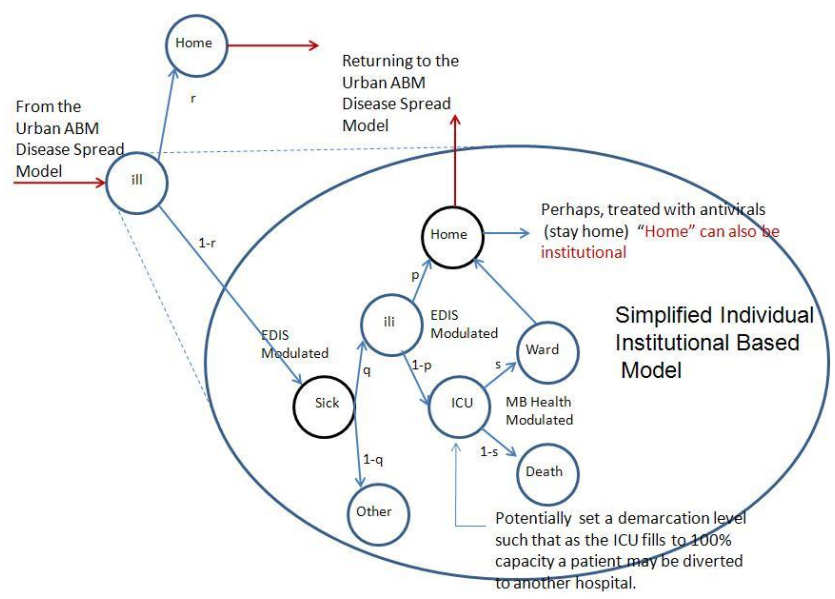

Fig. 3. The emergency department individual based model.

A statistic instrumented during the simulation was the number of agents arriving at individual hospital EDs. These surges are shown in Fig. 4 for the seven hospitals in the Winnipeg area.

\section{Hospital Loads}

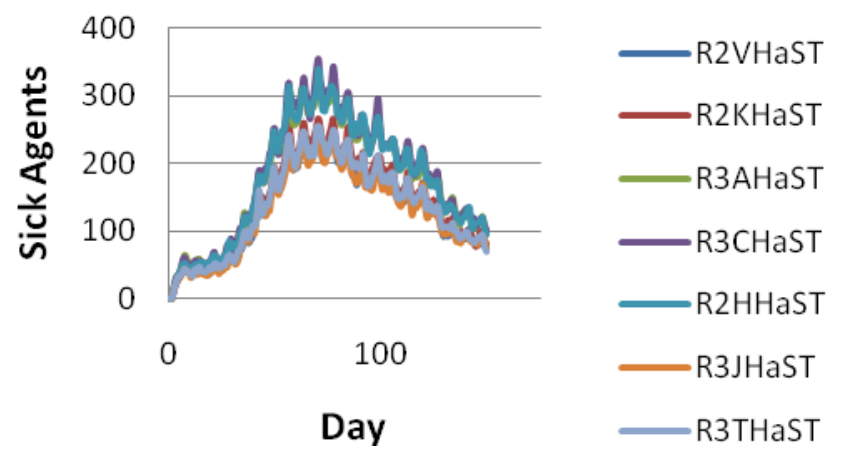

Fig. 4. Simulated Surges at Winnipeg Hospitals during an Outbreak 


\section{Hospital Loads}

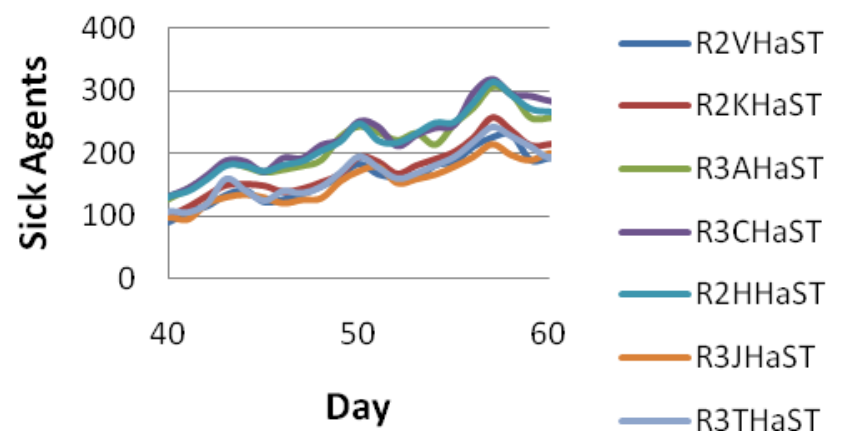

Fig. 5. Day of Week Variations at Winnipeg Hospitals during an Outbreak

Fig. 4 illustrates a disparity between various hospitals, corresponding to population density variations with the city. Fig. 5 illustrates the variation within weekly routines or schedules. These data are averaged over five runs. This averaging raises an interesting consideration from a policy perspective: as the data is averaged, it tends to become smoother and may tends to mask shortterm variations that may otherwise be present and important to note in the physical environment as well as the model.

\section{Results: Self redirection}

\section{B. 'Crowdinfoming' diversions}

Although expected, reduced surges at hospital EDs can reasonably be argued to facilitate improved treatment and service efficiencies during an outbreak. These outcomes illuminated a potential indirect intervention. The modelling initially planned for reactive hospital diversions, i.e. setting demarcation levels related to capacity to support diversion policy and redirection [20]. With recent interests in 'crowdsourcing' [16] and as a means to aid in biosurveillance of potential infection spread, the model was adapted to model the counterpoint of 'crowdsourcing', that being 'crowdinforming.' One of the fundamental tenets of 'crowdsourcing' is that the feedback loop needs to be closed, as information mined through crowdsourcing flows back to the crowd that generated it, presumably to accrue benefit.

At present, one trial hospital in the Winnipeg Regional Health Authority (WRHA) jurisdiction has an ED 'dashboard' in place, updating the number of patients waiting and their waiting times in terms of maximum wait at various triage levels. The dashboard is intended to inform those already in the waiting room of the anticipated wait before being seen. These dashboards are derived from the WRHA Electronic Data Information System (EDIS) which was recently rolled out across the WRHA hospitals. Our model develops this notion further and conjectures that in a reasonably short period of time, this data could be made publicly available through a web service, such that an individual would be able to query the hospital 'dashboards' from the WRHA site over the internet with any browser, be it mobile cellular or wired, or made available 
via a community call centre such as a 311 service whereby a patient would call and enquire about expected wait times.

For our modeling purposes, in this 'crowdinforming' scenario, a proactive decision is then made by an agent seeking treatment at an ED. In addition to the stochastic process of deciding upon going to an ED, each agent is also provided with estimates of wait times and the number of patients waiting. This data is available and easily instrumented within the ABM.

As an illustration of our weighted fair redirecting, we assumed four hospitals with waits of 1, 2, 3 and 4 hours with travel times of 60 minutes, 45 minutes, 30 minutes, and 15 minutes, coarsely extracted from topographical distances. An agent would create a roulette wheel to guide their decision once they have elected to go to an ED. The associated normalized probabilities associated with informed self redirection are shown in Table 1.

Table 1. Informed Emergency Department Self Redirect Probabilities

\begin{tabular}{|c|c|c|c|c|c|}
\hline & $\begin{array}{c}\text { ED } \\
1\end{array}$ & $\begin{array}{c}\text { ED } \\
2\end{array}$ & ED3 & ED4 & $\begin{array}{c}\text { Tot } \\
\text { al }\end{array}$ \\
\hline $\begin{array}{l}\text { Wait } \\
\text { Time } \\
\text { (hr) }\end{array}$ & 1 & 2 & 3 & 4 & \\
\hline $\begin{array}{l}\text { Travel } \\
\text { Time } \\
\text { (hr) }\end{array}$ & 1 & 0.75 & 0.5 & 0.25 & \\
\hline $\begin{array}{l}\text { Total } \\
\text { Time } \\
\text { (hr) }\end{array}$ & 2 & 2.75 & 3.5 & 4.25 & 12.5 \\
\hline 1/over & $1 / 2$ & $\begin{array}{l}1 / 2 . \\
75\end{array}$ & $\begin{array}{c}1 / 3 \\
5\end{array}$ & $\begin{array}{l}1 / 4 \\
25\end{array}$ & 1.38 \\
\hline $\begin{array}{l}\text { Probabili } \\
\text { ty }\end{array}$ & 0.36 & $\begin{array}{c}0.26 \\
4\end{array}$ & $\begin{array}{c}0.20 \\
7 \\
\end{array}$ & 0.17 & 1.0 \\
\hline
\end{tabular}

Table 1 combines estimates of travel time and wait times in an additive manner. Alternatively, it may be more appropriate to generate a total time as a linear combination weighted appropriately as in (1).

$$
\text { Total Time }=\square(\text { WaitingTime })+\square(\text { TravelTime })
$$

Using such a method, the agents make a probabilistic decision weighted by the least anticipated wait. As a consequence, the overall surge seen at hospitals is dampened by the behaviour of informed individuals. Modeling the 'crowdinformed' load-balancing results in surges at hospital as shown in Fig. 6, which was derived with $\square$ equal to zero. 


\section{Hospital Loads} w/redirection

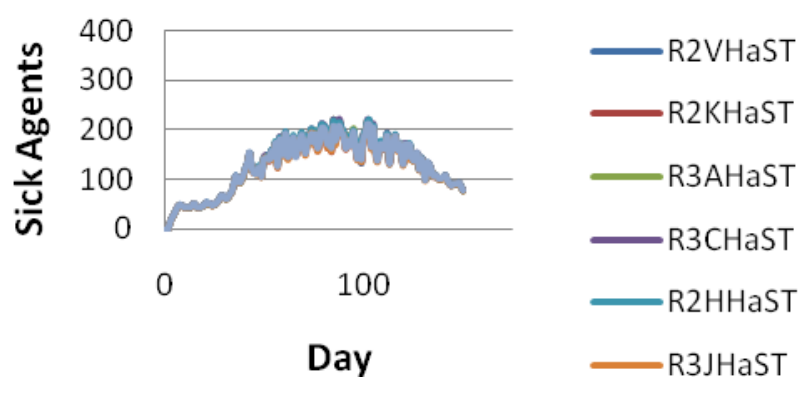

Fig. 6. Surges at Winnipeg Hospitals with 'crowdinforming'.

Crowdinforming appears to filter the simulated patient surges. A simple statistical measure associated with mean and variance indicates that the load balancing is statistically significant given our behavioural assumptions, where a known estimated wait time probabilistically influences a person's decision in attending a particular ED. The overall effect was expected to be that of a low pass filter smoothing out the peaks and valleys in both space and time. Fig. 6 clearly illustrates filtering in space as each ED roughly serves the same number of patients within a few percent. Filtering in time is expected to result from incorporation of an estimate of travel time as indicated in Equation 1. This simulation illustrates load balancing at the cost of greater variance in the patient load over time. This type of emergent behaviour inherently has greater information content than anticipated or expected results. While the simulation results are presently a coarse approximation of a policy implementation, they serve as an indication of possible trends and side effects.

As a further extension, the additions of temporary clinics were also modeled. The temporary clinics were modeled as being available provided sufficient staff resources existed to off-load EDs during a serious influenza outbreak. In this case, hospitals were augmented with temporary clinics and the public was informed of their location and services. Temporary clinics are modelled as being staffed with and providing a level of service similar to a hospital ED. In the following simulation, six temporary facilities were instantiated in highly populated postal code areas. Given the model of the information provided to the public, the consequent balancing of loads at hospital EDs and temporary clinics are seen to be balanced. Fig. 7 also demonstrates the effect of prioritizing travel in the decision-making process (Fig. 7 is derived with $\square$ equal to 0.5), with $80 \%$ of infected agents seeking treatment. In addition, acute patients are modeled as not presenting themselves at a clinic but rather deciding to directly attend an ED. An additional modification is that the temporary clinics are more heavily recommended once hospitals near capacity, or alternately that hospitals are preferred destinations until they are near capacity. 


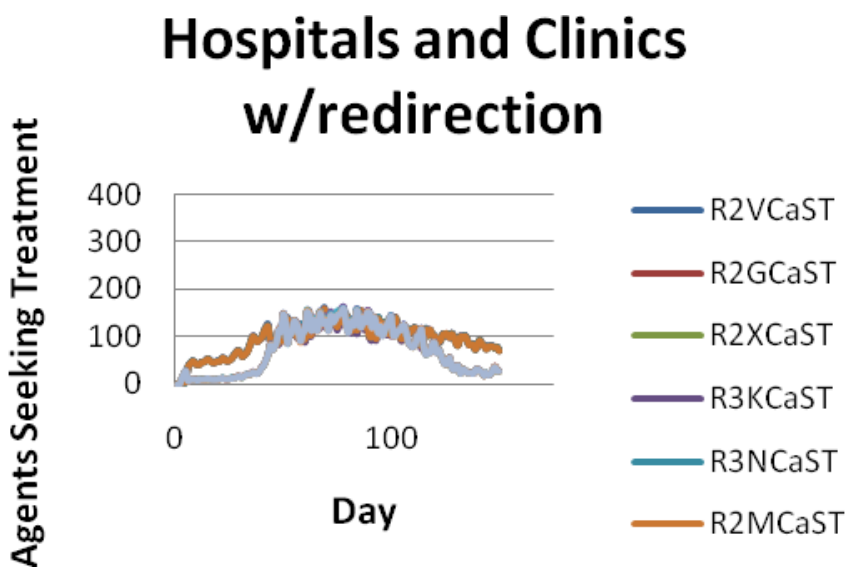

Fig. 7. Surges at Winnipeg Hospitals and Clinics with 'crowdinforming'.

An additional parameter of interest was the location of temporary clinics. For the simulation above, the clinics were located in central and highly populated postal code areas. The simulation allows for one to vary the location of clinics, such that they may better serve a community or region.

Fig. 8 illustrates a simulation run without averaging over multiple runs (which carries the effect of averaging over multiple days). Without averaging, the day to day variations are clearly apparent. If this variability were to be an actual consequence of the patient self redirection policy, it may be that the increase in day to day variation would not be a reasonable trade-off for a balanced load. It is these types of insights that ABM and what-if scenarios provide for policy makers a-priori to policy implementation.

\section{Hospital Loads w/redirection}

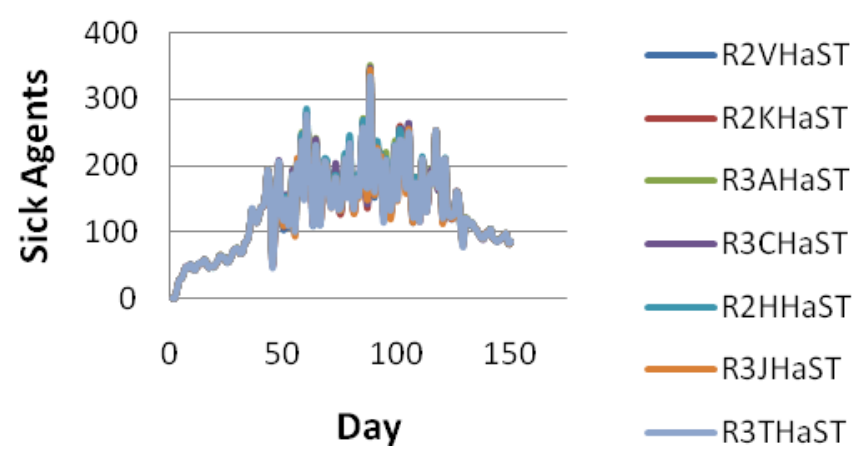

Fig. 8. Hospital Loads without averaging. 


\section{Discussion}

Although preliminary, this work represents one of the first modelled instances of the potential of 'crowdinforming' in providing a policymaker with a simulation to assist in public health decision support. In doing so, this work illustrated the role that an ABM can play in developing policy decision support systems. The model presented here illustrates how one specific intervention that of proactive information dissemination or 'crowdinforming' - can provide a degree of patient load balancing at EDs. A similar model was constructed corresponding to the scenario where sufficient public health resources existed for the deployment of temporary clinics. In the event of the temporary clinics, the model also allows for an investigation into their utilization where the public would be informed of their location and services.

The findings are somewhat self-evident in that the model for 'crowdinforming' contributed to load balancing at individual hospital EDs. The findings also indicate that the location of the clinics is reasonably important in off-loading EDs. The most significant limitations associated with research of this nature is that stochastic models of behaviour have to be estimated. Other shortcomings are associated with the access and usability of real data. These latter barriers are technological, while the former are theoretical. The validity of the underlying ABM framework is enhanced as the characterization of agents and their behaviours is improved and refined with additional real data. Consequently, barriers to access and usability of real data - whether technological or political - are limitations to the work. A more significant deficit in the model is that the deployment of temporary clinics assumes that the resources exist for staffing and treatment.

\section{Summary}

The work presented here allows a policy for patient directed redirection to be simulated, adding a qualitative assessment to a model that may otherwise be experiential or best-intent. This work is one of the first demonstrations of the 'crowdsourcing' intervention, and it demonstrates the role an $\mathrm{ABM}$ and similar technologies will continue to play in the future.

\section{Acknowledgments}

The authors acknowledge Maciej Borkowski, Marek Laskowski, and Bryan Demianyk for early developments of the ABM framework. The authors also acknowledge Manitoba Hydro for financial support.

\section{Correspondence:}

Bob McLeod

University of Manitoba

mcleod@ee.umanitoba.ca 


\section{References}

[1] Marshall A, Burns L. A Bayesian network hybrid model for representing accident and emergency waiting times. Proceedings of the IEEE Symposium on Computer-Based Medical Systems. June 2007; 91-96.

[2] Komashie A, Mousavi A. Modeling emergency departments using discrete event simulation techniques. Proceedings of the Winter Simulation Conference. Dec. 2005; 2681-2685.

[3] Patrick J, Puterman M. Reducing wait times through operations research: Optimizing the use of surge capacity. Healthcare Quarterly, 2008;11(3): 77-83.

[4] Khurma N, Bacioiu G, Pasek Z. Simulation-based verification of lean improvement for emergency room process. Proceedings of the Winter Simulation Conference. Dec 2008;14901499.

[5] McNulty T, Re-engineering Health Care: The Complexities of Organizational Transformation, New York, Oxford University Press, 2002.

[6] Kanagarajah A, Lindsay P, Miller A, Parker D. An exploration into the uses of agent-based modeling to improve quality of health care. International Conference on Complex Systems. June 2006; 1-10.

[7] Gunal M, Pidd M. Simulation modelling for performance measurement in healthcare. Proceedings of the Winter Simulation Conference, Dec. 2005; 2663 - 2668.

[8] Blachowicz D, Christiansen J, Ranginani A, Simunich K. How to determine future HER ROI: Agent-based modeling and simulation offers a new alternative to traditional techniques, J. Healthcare Information Management. Winter 2008;22(1):39-45.

[9] Gunal M, Pidd M. Understanding accident and emergency department performance using simulation. Proceedings of the $38^{\text {th }}$ conference on Winter simulation, 2006;446-452.

[10] Bonabeau E. Agent-based modeling: Methods and techniques for simulating human systems. Proceedings of the National Academy of Science. May 2002 [Online]. 99(Suppl 3), pp. 7280-7287. Available: http://www.pnas.org/content/99/suppl.3/7280.full\#xref-ref-31

[11] Epstein J. Modelling to contain pandemics. Nature. 2009:460;687.

[12] Hupert N, Xiong W, Mushlin A. The virtue of virtuality: The promise of agent-based epidemic modeling," Translational Research. 2008:151(6):273-274.

[13] Epstein J. Artificial society: Getting clues on how a pandemic might happen by creating a huge model of the United States, The Brookings Institution. [Online]. Available: www.brookings.edu/interviews/2008/0402_agent_based_epstein.aspx.

[14] Merler S, Ajelli M, Jurman G, Furlanello C, Rizzo C., Bella A, Massari M, Ciofi degli Atti M. Modeling influenza pandemic in Italy: An individual-based approach. The 2007 intermediate conference of the Italian Statistical Society. June 2007. Available http://www.sis-statistica.it/files/pdf/atti/SIS\%202007\%20Venezia\%20intermedio_121131.pdf

[15] M. Borkowski M, Podaima B, McLeod R., Epidemic modeling with discrete space scheduled walkers: Possible extensions to HIV/AIDS," BMC Public Health, vol. 9 (Suppl 1): S14, 2009. [Online\}. Available: doi:10.1186/1471-2458-9-S1-S14.

[16] Song, C, Qu Z, Blumm N, Barabási A. Limits of Predictability in Human Mobility, Science, 2010 327(5968);1018-1021. 
[17] Howe J, The Rise of Crowdsourcing. Wired. June 2006, http://www.wired.com/wired/archive/14.06/crowds.html.

[18] Laskowski M, Borkowski M, Demianyk B, Friesen M, McLeod R. The utility of agentbased models for healthcare applications," The Second IASTED International Conference on Telehealth and Assistive Technology, Cambridge, MA, Nov. 2009.

[19] Laskowski M, McLeod R,Friesen M, Podaima B, Alfa A., Models of emergency departments for reducing patient waiting times. PLoS ONE,. 2009;4(70: e6127. [Online]. Available: doi:10.1371/journal.pone.0006127, 2009.

[20] Mukhi S, Laskowski M, Agent-based simulation of emergency departments with patient diversion. Electronic Healthcare, D. Weerasinghe, Ed. Berlin: Springer. 2009;25-37. 